\title{
Grasp modelling with a biomechanical model of the hand
}

\author{
Joaquín L. Sancho-Bru
}

Department d'Enginyeria Mecànica i Construcció, Universitat Jaume I, Castelló de la Plana, Spain

Campus de Riu Sec. Avinguda Vicent Sos Baynat s/n. 12071 Castelló de la Plana (Spain).E-mail: sancho@emc.uji.es

Marta C. Mora

Department d'Enginyeria Mecànica i Construcció, Universitat Jaume I, Castelló de la Plana, Spain

\section{Beatriz E. León}

Department d'Enginyeria i Ciència dels Computadors, Universitat Jaume I, Castelló de la Plana, Spain

\section{Antonio Pérez-González}

Department d'Enginyeria Mecànica i Construcció, Universitat Jaume I, Castelló de la Plana, Spain

José L. Iserte

Department d'Enginyeria Mecànica i Construcció, Universitat Jaume I, Castelló de la Plana, Spain

\section{Antonio Morales}

Department d'Enginyeria i Ciència dels Computadors, Universitat Jaume I, Castelló de la Plana, Spain 


\section{Grasp modelling with a biomechanical model of the hand}

The use of a biomechanical model for human grasp modelling is presented. A previously validated biomechanical model of the hand has been used. The equilibrium of the grasped object has been added to the model through the consideration of a soft contact model. A grasping posture generation algorithm has been also incorporated to the model. All the geometry has been represented using a spherical extension of polytopes (s-topes) for efficient collision detection. The model has been used to simulate an experiment in which a subject was asked to grasp two cylinders of different diameter and weight. Different objective functions have been checked to solve the indeterminate problem. The normal finger forces estimated by the model have been compared to the ones experimentally measured. The popular objective function sum of the squared muscle stresses has been shown not suitable for the grasping simulation, requiring at least being complemented by task dependent grasp quality measures.

Keywords: grasp; biomechanical model; finger force estimation

\section{Introduction}

To date, many biomechanical models of the hand have been developed with the aim of providing a tool for studying problems that cannot be directly analysed on humans or that have an experimental cost that is too high; e.g., the study of new alternatives for restoring hand pathologies.

First models were very simplified two-dimensional models of a single finger, allowing only flexion-extension movements. They were used to explain the function of different anatomical elements (Leijnse et al. 1992; Leijnse and Kalker 1995; Spoor and Landsmeer 1976; Spoor 1983; Storace and Wolf 1979, 1982; Thomas et al. 1968), the movement coordination of the interphalangeal joints (Buchner et al. 1988; Lee and Rim 1990), to study the causes and effects of hand pathologies (Smith et al. 1964; Storace and Wolf 1979, 1982) or even to obtain approximate values for the articular forces for testing prosthetic designs (Weightman and Amis 1982). By the year 2000, few attempts 
of developing a three-dimensional model were performed (Biryukova and Yourovskaya 1994; Casolo and Lorenzi 1994; Chao and An 1978; Chao et al. 1976; Esteki and Giurintano et al. 1995; Mansour 1997; Mansour et al. 1994; Valero-Cuevas et al. 1998). These models allowed the study of more complex movements, but still none of them modelled the complete hand.

Recent models are more complete but do not differ much from the ones developed before 2000 (Fok and Chou 2010; Kamper et al. 2006; Kurita et al. 2009; Lee et al. 2008a, 2008b; Qiu et al. 2009; Roloff et al. 2006; Sancho-Bru et al. 2001, 2003a, 2003b, 2008; Valero-Cuevas 2000, 2005; Valero-Cuevas et al. 2000; Vigouroux et al. 2006; Wu et al. 2010). Briefly, the hand kinematics is modelled using the concept of the instantaneous centre of rotation. Thus, all these works use fixed axes of rotation; depending on the joint, one or two axes of rotation are considered. Tendons, operated by muscles, control the kinematics of the hand skeletal chains. To model the action of tendons crossing a joint, the models consider the ideal case of a non-friction belt around a pulley. The muscle behaviour is modelled in most of the works in the literature by using a simple Hill's model that allows the consideration of the three main parameters, i.e., the muscle activation level, and the variation of the maximum deliverable muscle force with the muscle length and the muscle contraction velocity. Finally, the dynamic equilibrium equations on the skeletal chains are obtained, leading to an indeterminate system of equations, with more unknowns (muscle and tendon forces) than available equations. Inequality constraints taking into account the maximal forces that may be delivered by each muscle and that tendons cannot support compressive forces are considered as well. The problem is solved by minimising some cost function. Different cost functions have been investigated, being the most popular the sum of the squared 
muscle stresses, which has been related to the maximisation of fatigue resistance (Crowninshield and Brand 1981).

Such models have been used in the literature to estimate the muscle forces required to counteract given external forces on the hand while performing given movements. To do that, they consider movements and contact zones and forces that had to be experimentally measured.

Most of the effort in hand biomechanics until now has been focused on appropriately modelling the different hand components (kinematics, muscles, tendons, etc.). But little effort has been made on the formulation of the grasping problem when using a biomechanical model. In this sense, many limitations persist. Current models do not allow the estimation of the contact information required by a biomechanical model for simulating the grasping of objects.

In this sense, although the human hand is obviously more complex than robot hands, the methods used in robotics might be raised up to study the human grasp by considering the hand as the human end-effector.

A robot should be able to locate the object and then grasp it. A contact model is defined to determine the forces or torques that the robot manipulator must exert on the contact areas. The more common contact models used in robotic grasping are the point contacts with and without friction and the soft-finger contacts (Roa Garzón 2009). Point contact models, also named rigid-body contact models, assume rigid-body models for the hand and the grasped object while the soft-finger contact models, also called compliant or regularised models, assume that the hand is a deformable element grasping a rigid body (Kao et al. 2008). The soft contact model allows the finger to apply an additional torsional moment with respect to the normal at the contact point (Ciocarlie et al. 2005, 2007; Howe et al. 1988; Howe and Cutkosky 1996; Kao and Cutkosky 1992; 
Kao and Yang 2004). Unlike robot hands, human fingers conform to the grasped object shape, then only the soft-finger contact might be applied to the study of the human grasp.

In this work we present the use of a biomechanical model for simulating the human grasp by taking into account the equilibrium not only of the grasping hand but also of the grasped object, through the consideration of a soft contact model. In particular, the objective function required to solve the indeterminate problem is investigated.

\section{Materials and methods}

A previously validated 3D scalable biomechanical model of the complete hand (SanchoBru et al. 2001, 2003a, 2003b, 2008) has been used to incorporate the grasping capabilities. The original model required the posture of the hand, the contact points and the contact forces as input data. With these data, the model allowed the estimation of the muscle forces required to counteract the given external forces on the hand while performing the given movements. For such goal, the model considered the popular criterion of maximizing the endurance in order to solve the indeterminate problem of finding the muscle forces.

The model has been modified to tackle the grasping problem, as it is described in section 2.1. Briefly:

- A grasping posture generation algorithm has been incorporated to the model to automatically generate the hand posture during grasping, along with the contact points and normals required by the original model.

- The magnitudes of the contact forces are solved together with the muscle forces 
by means of adding to the original model the equilibrium equations of the grasped object. For that purpose, a soft contact model between the grasping hand and the grasped object has been used.

The validity of the model has been checked by reproducing a grasping experiment described in section 2.2. In particular, the validity of the criterion of maximizing the endurance to solve the indeterminate problem is investigated.

\subsection{Model description}

\subsubsection{Musculoskeletal model}

The biomechanical model uses the anthropometric parameters hand length (HL) and hand breadth (HB) to scale all its components (Fig. 1).

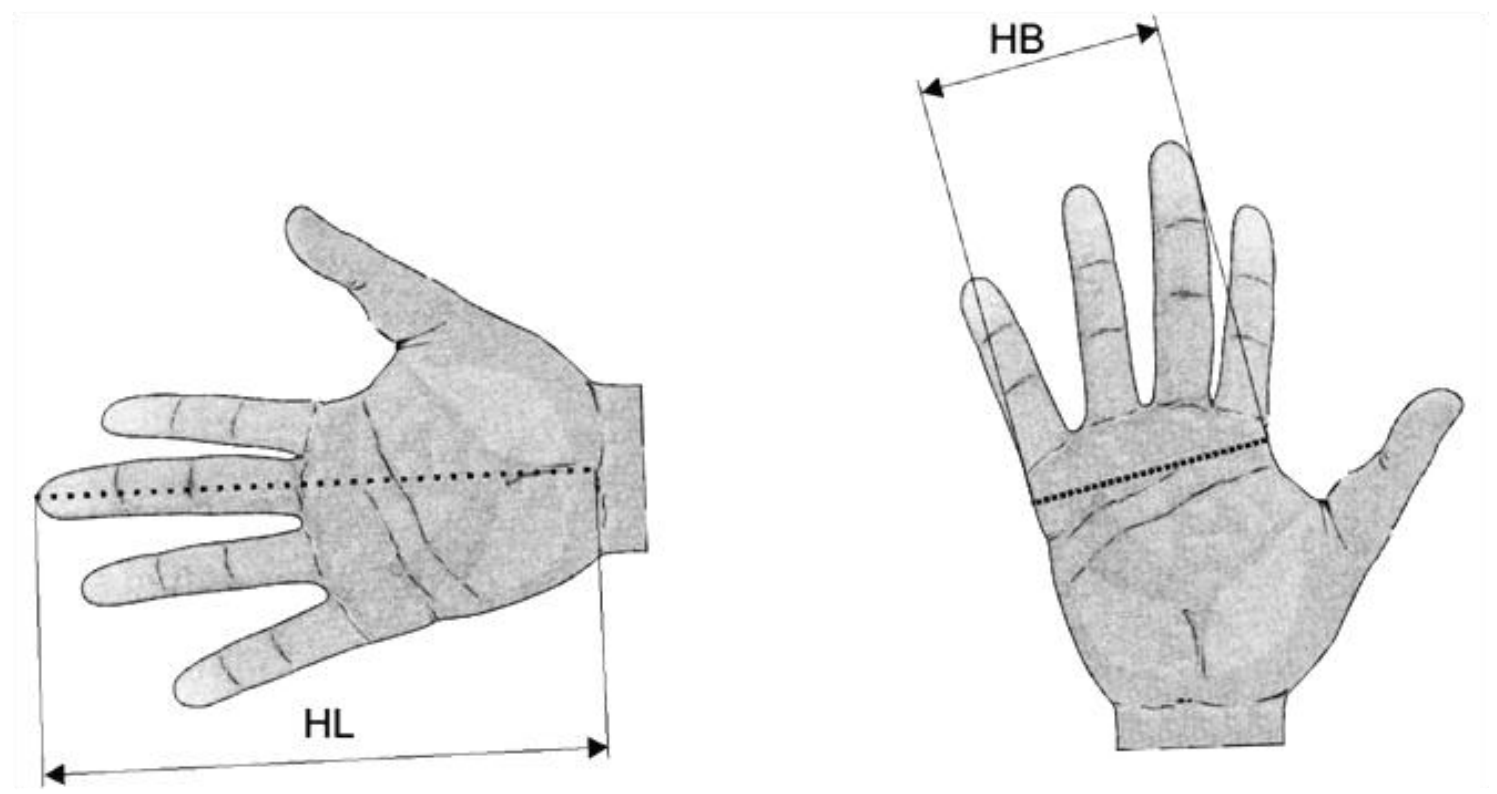

Figure 1: Parameters used to scale the model: HL (hand length) and HB (hand breadth)

Kinematic model. The hand model considers 23 degrees of freedom (DOF) selected to realistically simulate the hand movements. The hand has been modelled as five skeletal open chains of rigid bodies (the bones) connected to the carpus through different joints. 
Proximal and distal interphalangeal joints (PIP and DIP) of the fingers and the interphalangeal joint (IP) of the thumb are of trochlear type, allowing only flexionextension movements (Brand and Hollister 1992). They have been modelled as hinge joints.

All metacarpophalangeal joints (MCP) are of condilar type, allowing both flexion-extension and abduction-adduction movements (Brand and Hollister 1992). The carpometacarpal joint (CMC) of the thumb is a saddle joint, also allowing flexionextension and abduction-adduction movements (Brand and Hollister 1992). All these joints have been modelled as universal joints.

Finally, the model considers the palm arching (very important for grasping) through the model of the little and ring CMC joints. These joints are of arthrodial type, with a very limited movement range (Kapandji 1998). They have been modelled as hinge joints.

Data for the axes location and orientation were obtained from (An et al. 1979; Buchholz et al. 1992; Hollister et al. 1995). These data along with the segment lengths' data were appropriately scaled with respect to the parameters HB and HL (Sancho-Bru et al. 2003b).

Musculotendinous model. Muscles have been modelled using a simple Hill's threecomponent model (Hill 1938) that takes into account the muscle activation level $(\alpha)$ and the force-length and force-velocity relationships, as well as the different index of architecture of muscles. The model considers a contractile element (CE), which is the basic component that generates force, a parallel elastic element (PEE), which is responsible for the passive force generated by the muscle when it is stretched, and a series elastic element (SEE), the muscle tendon unit, which has been considered to be inextensible (Fig. 2). 


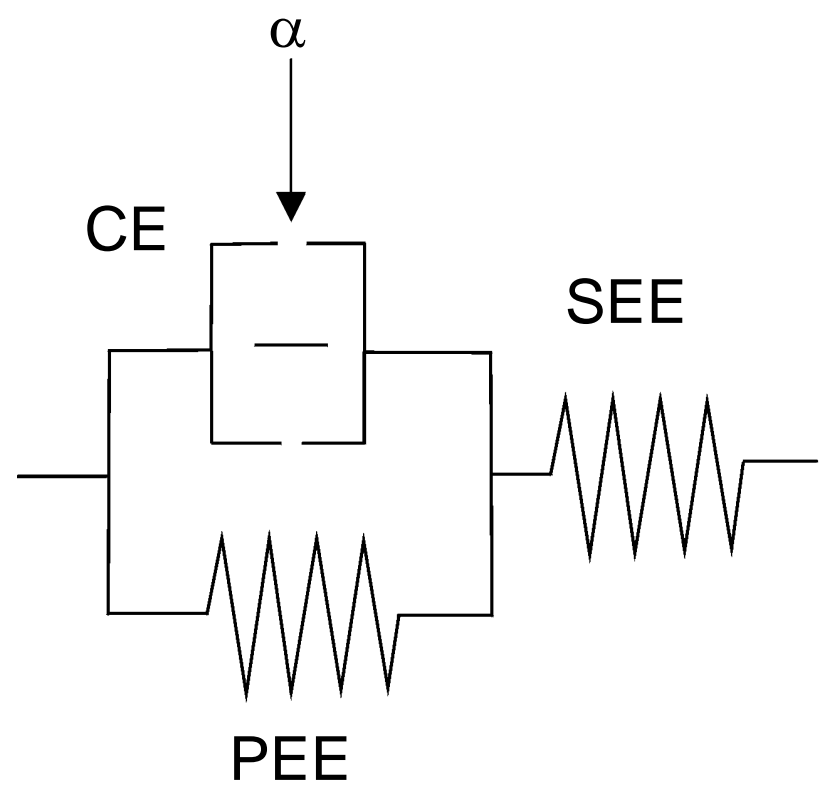

Figure 2. Hill's muscle model

The maximum force a muscle can exert in optimal conditions is proportional to its physiological cross-sectional area (PCSA):

$$
F_{\text {max }}=P C S A \cdot S_{\text {max }},
$$

where $S_{\text {max }}$ is the maximum stress the muscle can bear (An et al. 1991).

As the strain of tendons is insignificant for the magnitude of the forces developed by the muscles (Goldstein et al. 1987), the SEE has been considered to be inextensible. Then, the force the muscle exerts $(F)$ can be written as:

$$
F=F_{\text {max }}\left(F_{C E}+F_{P E E}\right),
$$

where $F_{C E}$ and $F_{P E E}$ are the normalised forces delivered by the $\mathrm{CE}$ and PEE, respectively. The force exerted by the muscle can be decomposed into an active force corresponding to the $\mathrm{CE}$ and a passive force corresponding to the PEE. The force delivered by the $\mathrm{CE}$ is related to the muscle architecture and is a function of the muscle 
length $l_{C E}$, the contraction velocity $v_{C E}$, and the muscle activation level $\alpha$ (from 0 to 1 ), which is controlled by the central nervous system (Kaufman et al. 1991):

$$
F_{C E}=\alpha \cdot F_{l}\left(l_{C E}\right) \cdot F_{v}\left(v_{C E}\right),
$$

where $F_{l}$ and $F_{v}$ are the non-dimensional force-length and force-velocity relationships, that have been modelled using the expressions proposed by Kaufman et al. (1991) and Hatze (1981), respectively.

The force generated by the PEE is a function only of its length, and has been modelled considering an exponential relationship (Kaufman et al. 1991; Lee and Rim 1990).

The scalability of the muscular action is achieved by scaling the PCSA of the muscles with respect to the product of the hand length and hand breadth parameters (Sancho-Bru et al. 2008) from its value for a reference hand size:

$$
\frac{P C S A(H L, H B)}{P C S A(\overline{H L}, \overline{H B})}=1+0.013 \cdot(H L \cdot H B-\overline{H L} \cdot \overline{H B})
$$

The muscles considered on each skeletal chain are listed in Table 1. PCSA data for index finger muscles have been taken from Valero-Cuevas et al. (1998); data for the remaining muscles have been obtained from Brand and Hollister (1992). The muscle stress limit $\left(S_{\max }\right)$ has been obtained from Zajac (1989). The remaining required parameters to establish the force-length and force-velocity relationships have been obtained from Lee and Rim (1990), Lemay and Crago (1996), Jacobson et al. (1992) and Kaufman et al. (1991). 
Table 1. Muscles modelled on each skeletal chain (acronyms in the nomenclature section)

\begin{tabular}{ccccc}
\hline Index & Medial & Ring & Little & Thumb \\
\hline $1^{\text {st }} \mathrm{FP}$ & $2^{\text {nd }} \mathrm{FP}$ & $3^{\text {rd }} \mathrm{FP}$ & $4^{\text {th }} \mathrm{FP}$ & APB \\
$1^{\text {st }} \mathrm{FS}$ & $2^{\text {nd }} \mathrm{FS}$ & $3^{\text {rd }} \mathrm{FS}$ & $4^{\text {th }} \mathrm{FS}$ & FPB \\
$1^{\text {st }} \mathrm{EDC}+\mathrm{EI}$ & $2^{\text {nd }} \mathrm{EDC}$ & $3^{\text {rd }} \mathrm{EDC}$ & $\mathrm{EDQ}$ & OPP \\
$1^{\text {st }} \mathrm{LU}$ & $2^{\text {nd }} \mathrm{LU}$ & $3^{\text {rd }} \mathrm{LU}$ & $4^{\text {th }} \mathrm{LU}$ & $\mathrm{ADD}$ \\
$1^{\text {st }} \mathrm{DI}$ & $2^{\text {nd }} \mathrm{DI}$ & $4^{\text {th }} \mathrm{DI}$ & $3^{\text {rd }} \mathrm{VI}$ & $1^{\text {st }} \mathrm{DI}$ \\
$1^{\text {st }} \mathrm{VI}$ & $3^{\text {rd }} \mathrm{DI}$ & $2^{\text {nd }} \mathrm{VI}$ & FDQ & APL \\
& & & ADQ & EPB \\
& & & & FPL \\
& & & & EPL \\
\hline
\end{tabular}

Most of the muscles do not act directly on the bones, but through the force transmitted to the tendons. To model the tendon action crossing the joints, straight lines connecting 2 points have been considered, one fixed with respect to the proximal bone and the other one with respect to the distal bone (Fig. 3a). This approximation has been found to be close enough to the behaviour of all tendons with the exception of extensors (An et al. 1979), for which Landsmeer's model I has been considered (Fig. 3b).

a)

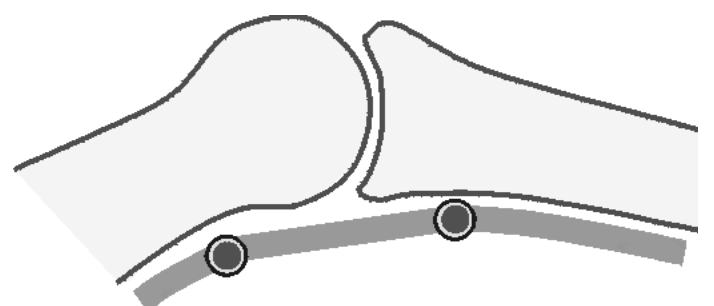

b)

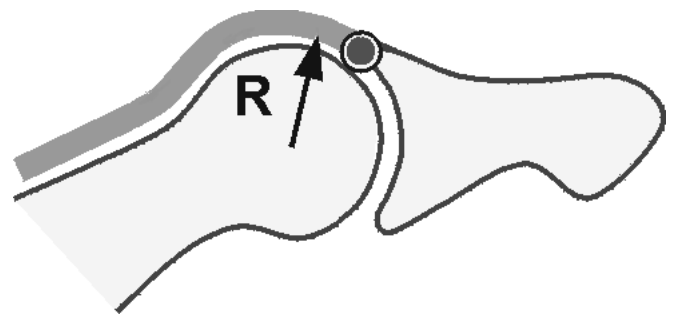

Figure 3. Models for the tendons crossing the joints (Sancho-Bru et al. 2001): a) Straight lines; b) Landsmeer's model I. 
The extensor hood mechanisms of the fingers are modelled as a deformable tendon net (Sancho-Bru et al. 2003b), in which the appropriate force balances have been considered.

The data for the points defining the tendon paths have been obtained from An et al. (1979), and have been also scaled with respect to HB and HL (Sancho-Bru et al. 2003b).

\subsubsection{Grasping posture generation}

In order to generate grasp postures automatically, we used a grasping algorithm based on that of Choi (2008). This algorithm uses a function to automatically generate a natural grasping motion path of the hand model from a fully opened state to a clenched one. The goal is to find contacts between the surface hand skin and the object surface while rotating the joint angles of the fingers. Care has to be taken in properly choosing the rotation rate of the finger joints, as it affects the final posture prediction. Based on the results from Choi (2008), we have used a variable rotation algorithm, by describing the rotations of all joints at observation-based rates. The rotation rate is defined by the difference between the measured angles of the most fully opened state and the tentative clenched one.

In order to generate the grasp, a contact model is required. We need to check whether the surface skin model makes contact with the surface of the object model. In reality, the surface of a hand is deformed when making contact with the object. Generally, this deformation has a non-linear elastic behaviour, and it could be simulated using finite element analyses. Nonetheless, this would need a long execution time that we considered unacceptable. Therefore, we considered a simple geometric collisiondetection algorithm based on the one used by Endo et al. (2007). The algorithm allows 
the penetration of the surface skin model and the object model. This penetration is limited by a tolerance that relates to the hand stiffness at each contact region. At this first approximation to the grasp problem, we considered only grasps involving contact at the fingertips. A maximum penetration of $3 \mathrm{~mm}$ has been considered for all fingertips.

The distances between the points on the skin surface and the object are calculated while each joint rotate according to the specific joint rotation algorithm. When the distance between the skin surface points and the object reaches the given maximum penetration tolerance, the contact is achieved and the joint rotation ends. When the distal segments of all four fingers make contact with the object, the grasping simulation terminates.

In order to perform these calculations in an efficient way, the geometry of the hand surface and the grasped object have been modelled using the spherical extension of polytopes (s-topes). This graphical representation has been successfully used previously in robotics (Bernabeu and Tornero 2002), allowing a fast and efficient collision detection between the grasping hand and the grasped object while showing a sufficient level of realism (Fig. 4). The collision detection is performed by calculating the minimum distance between s-topes, based on the Gilbert-Johnson-Keerthi algorithm (Gilbert et al. 1988). The algorithm also calculates the minimum distance points that define the normal direction to the contact surface. 


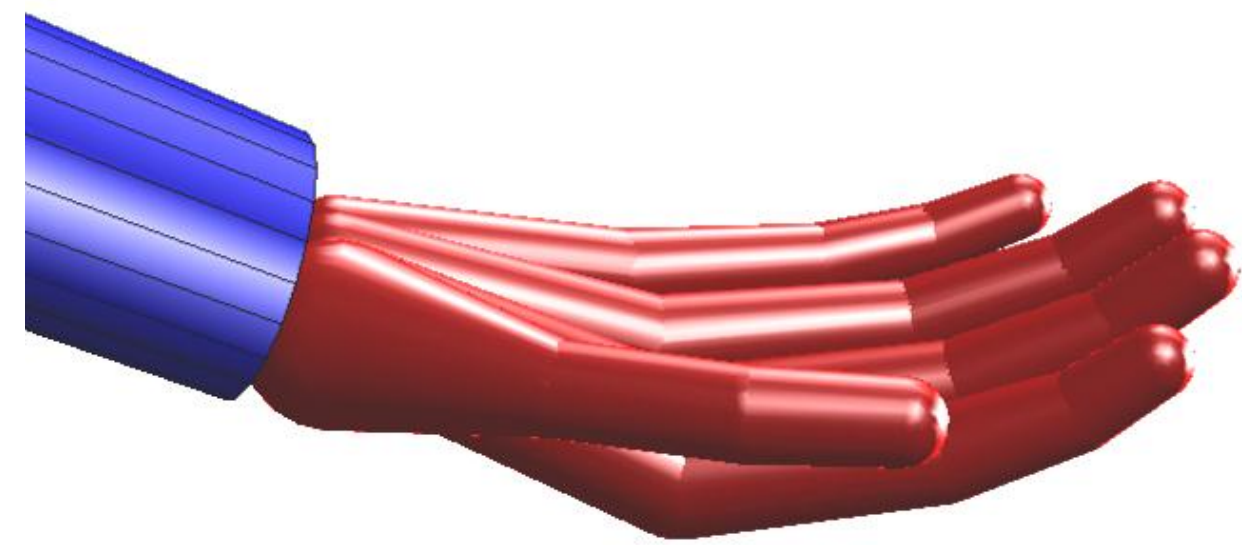

Figure 4. External geometrical representation of the hand with s-topes

\subsubsection{Soft contact model}

The contact forces between the object and the hand have to be considered when dealing with the estimation of the muscle forces required for grasping an object. Unlike what happens with robots, real human fingers conform to the grasped object shape. As the contact finger surface is deformable, the contact does not occur at just one point but over some finite area that increases as the normal forces increase. Due to this effect, in addition to the normal force and tangential force due to friction, human finger contact may support frictional torsional moments with respect to the normal at the contact point. This clearly shows that the consideration of rigid contacts, commonly used in robotics, is not appropriate for its use in studying the human grasp, and a soft contact model has to be used. Most objects manipulated by human hands are much stiffer than human hands and, therefore, it is reasonable for those cases to consider the grasped objects as rigid bodies and the hand as a deformable body.

In this case, a soft contact model based on that of (Ciocarlie et al. 2005) has been used. Friction constraints are derived based on general expressions for non-planar contacts of elastic bodies, taking into account the local geometry and structure of the 
objects in contact. The following approximation has been used to express the constraint relating the magnitudes of frictional force $\left(f_{t}\right)$ and moment $\left(\tau_{n}\right)$ :

$$
f_{t}^{2}+\frac{\tau_{n}^{2}}{e_{n}^{2}} \leq \mu^{2} \cdot P^{2}
$$

where $P$ is the total load applied in the direction of the contact normal, $\mu$ is the friction coefficient and $e_{n}$ is called the eccentricity parameter (height of the ellipsoid described by Eq. 6). Considering a Winkler elastic foundation (Johnson 1985) of depth $h$ and elastic modulus $K$, the eccentricity parameter is given by:

$$
e_{n}=\frac{8}{15} \cdot \sqrt{a \cdot b}
$$

where $a$ and $b$ can be calculated from the relative radii of curvature $R$ ' and $R$ '” of the objects in contact and the compression $\delta$ of the elastic layer:

$$
a=\sqrt{2 \cdot \delta \cdot R^{\prime}} ; b=\sqrt{2 \cdot \delta \cdot R^{\prime \prime}} ; \delta=\sqrt{\frac{P \cdot h}{K \cdot \pi \cdot\left(R^{\prime} \cdot R^{\prime \prime}\right)^{1 / 2}}},
$$

The values of $\mu$ and $K$ have been obtained from Savescu et al. 2008) and Hajian and Howe (1997), respectively.

\subsubsection{Problem solving and neuromuscular control}

The problem to be solved is to find the muscle forces required to grasp the object. That entails to account for the equilibrium of the grasping hand and the grasped object. It is an inverse dynamics problem. 
The dynamics equations of the open chain of rigid bodies have been derived using the Lagrange method (García de Jalón and Bayo 1994). For a system with $m$ generalised co-ordinates $q_{k}$, this equation is expressed as:

$$
\frac{d}{d t} \frac{\partial L}{\partial \dot{q}_{k}}-\frac{\partial L}{\partial q_{k}}=Q_{k}^{n c} \quad k=1, \ldots, m,
$$

where $L$ is the Lagrangian function and $Q_{k}^{n c}$ are the generalised non-conservative forces. The generalised coordinates have been considered coincident with the system DOF $(m=23)$.

Eq. 8 together with the force balances of the tendon nets make up the equilibrium equations of the grasping hand (49 equations). The equilibrium of the grasped object is defined by six more equations. A total of 55 equations with 99 unknowns (muscle and tendon forces and contact forces and moments) form the final grasping mathematical problem, along with the inequalities given by the muscle model (lower and upper bounds of muscle forces and lower bounds of tendon forces) and the soft contact model (one inequality by contact point). There is not a unique combination of muscular efforts that satisfy the equilibrium constraints. To solve the problem, a criterion chosen by the central nervous system to determine the muscle action control must be introduced.

The most commonly used criterion in the literature is the maximisation of the endurance (Crowninshield and Brand 1981), through the minimisation of the non-linear objective function

$$
O B J=\sum_{i=1}^{l}\left(\frac{F_{i}}{P C S A_{i}}\right)^{n}
$$


with $n$ between 2.0 and 4.0 (being 2.0 the most used), and being $l$ the number of muscles $(l=34$ in our model). The validity of this criterion for the grasping simulation will be checked in this work.

The MATLAB system and its optimisation toolbox (version R2008b) have been used to implement the model.

\subsection{Validation experiment}

The validity of the model was analysed through the simulation of grasping cylindrical objects. An experiment was designed in which a female subject (age 32, height $1.61 \mathrm{~m}$, weight $68 \mathrm{~kg}$, HB $71 \mathrm{~mm}$, HL $163 \mathrm{~mm}$ ), appropriately instrumented, was asked to grasp alternatively two cylinders of different size and weight and hold them with their axes in vertical orientation (gravity direction).

The subject was seated at a table which height was adjusted so that the subject's elbow coincided with the table height. The subject's arm was lying on the table in a relaxed posture, with the hand placed about $15 \mathrm{~cm}$ away from the cylinder to be grasped. The subject was asked to grasp each cylinder with her fingertips and hold it at a fixed height while keeping it in vertical orientation, during two to three seconds approximately, and then return it to its initial location.

First, the subject's hand was instrumented with the Cyberglove $\AA$ system (Cyberglove, Immersion Corp.) to register hand posture data. The system was appropriately calibrated (Mora et al. 2011). The subject repeated the action three times for training without data registration, and five more times with posture data registration (Fig. 5, left). Second, subject's hand was instrumented with the Finger TPS ${ }^{\circledR}$ system (Pressure Profile Systems, Inc) at her fingertips to register finger force data. After the calibration of the system was performed, the subject repeated the action three times for 
training without data registration, and five more times with force data registration (Fig.

5 , right).

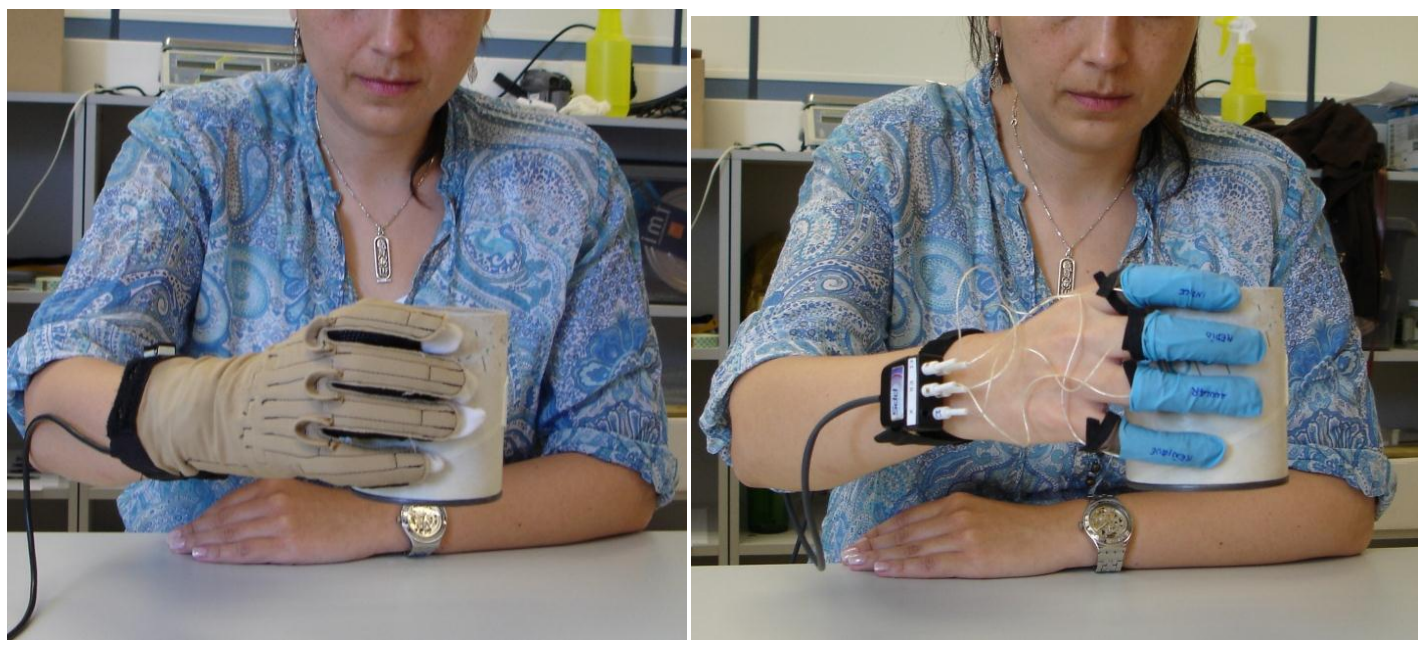

Figure 5. The subject is holding the lighter cylinder (cylinder 1) of the experiment. Left, hand instrumented for posture data registration. Right, hand instrumented for finger force data registration.

This procedure was performed twice: first for a cylinder of $0.401 \mathrm{~kg}$ of weight and $64 \mathrm{~mm}$ of diameter (cylinder 1) and second for a cylinder of $0.04 \mathrm{~kg}$ of weight and $82 \mathrm{~mm}$ of diameter (cylinder 2).

The model was used to simulate the grasping of both cylinders. The simulation only considered the static case of holding the cylinders at a fixed height. To perform the simulation, the subject's hand data (HL and HB) and the object data (weight and diameter) were input to the model, along with the most open posture (MOP) of the hand and the experimental grasping posture (EGP) registered with the Cyberglove $®$. Figure 6 shows the block diagrams of the original biomechanical model and the model proposed in this paper. So far, the registered postures are required to generate the rotation angle rates that are used by the grasping posture generation algorithm to obtain the predicted final grasping posture, the contact points and contact normal directions. This information is used by the optimization algorithm to obtain the contact forces and the muscle forces required for the equilibrium of the grasped cylinder and the grasping 
hand. The authors are already working on developing a neural network able to automatically obtain both postures and avoid the use of registered data. The data reported by Savescu et al. (2008) were used to select the appropriate friction coefficient (0.8) between the hand and the cylinder material being grasped.
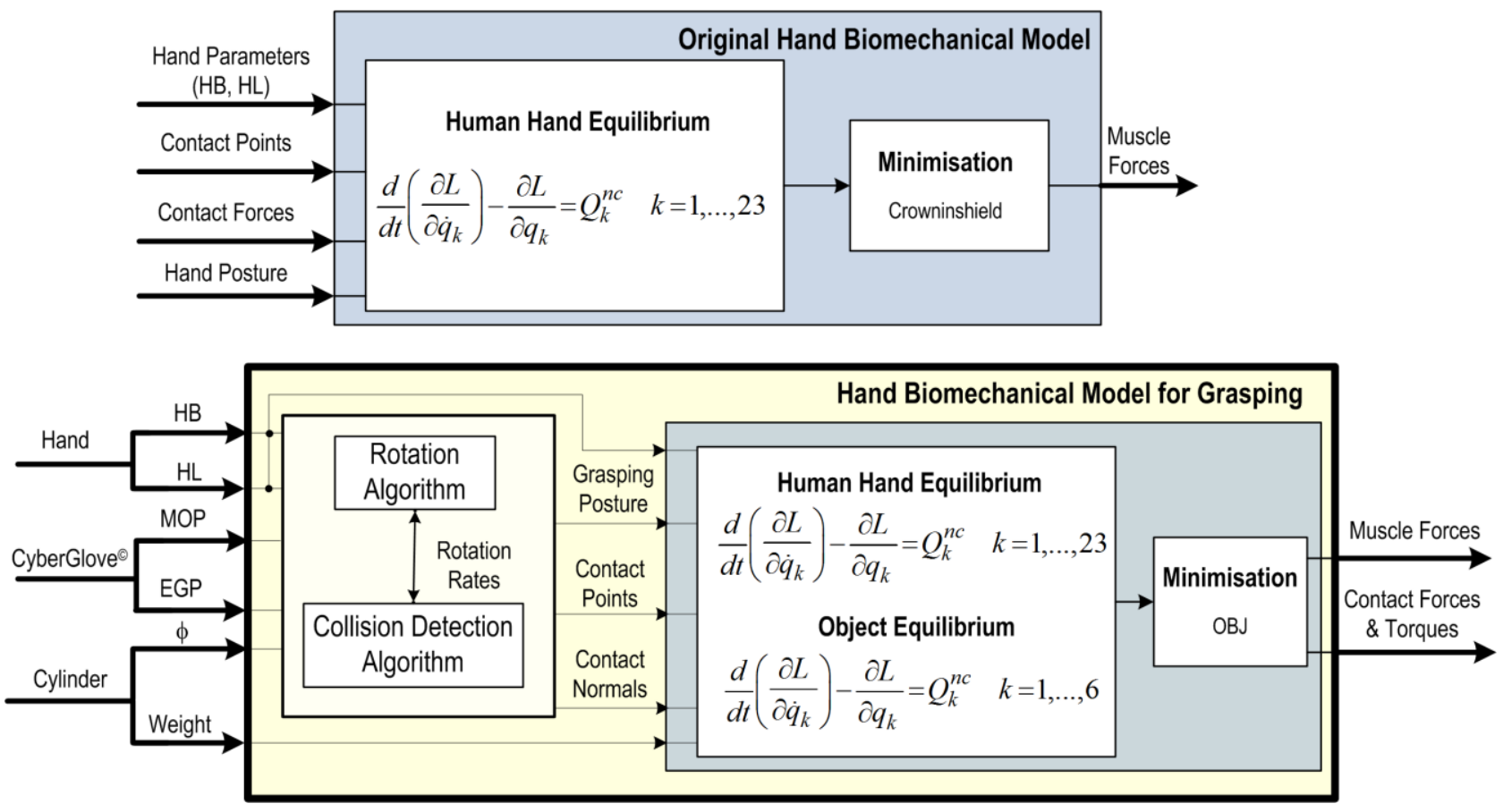

Figure 6. Block diagrams of the original biomechanical model and the proposed model for grasping.

The results of the simulation of grasping both cylinders were the grasping postures, the contact points, the contact normal directions, the contact finger forces and torques, and the muscle force distribution. The normal finger forces estimated by the model were compared to the ones registered with the Finger TPS $®$ system. To investigate how the model could be improved to achieve better results, the simulation of grasping both cylinders was performed under four different modifications of the model, described in the results section. 


\section{Results and discussion}

The hand movement pattern during the experiment can be observed in figure 7 . This figure shows the joint angles registered by the Cyberglove ${ }^{\circledR}$ system in one of the repetitions for cylinders 1 and 2. The hand starts from a relaxed posture. Just before grasping the cylinder, the hand gets open, which is seen mainly as an extension and abduction of MCP joints. The grasping is then achieved basically by means of the flexion of the different joints. Once the object is grasped, the joint angles registered during the static hold of the cylinders remain quite constant. 


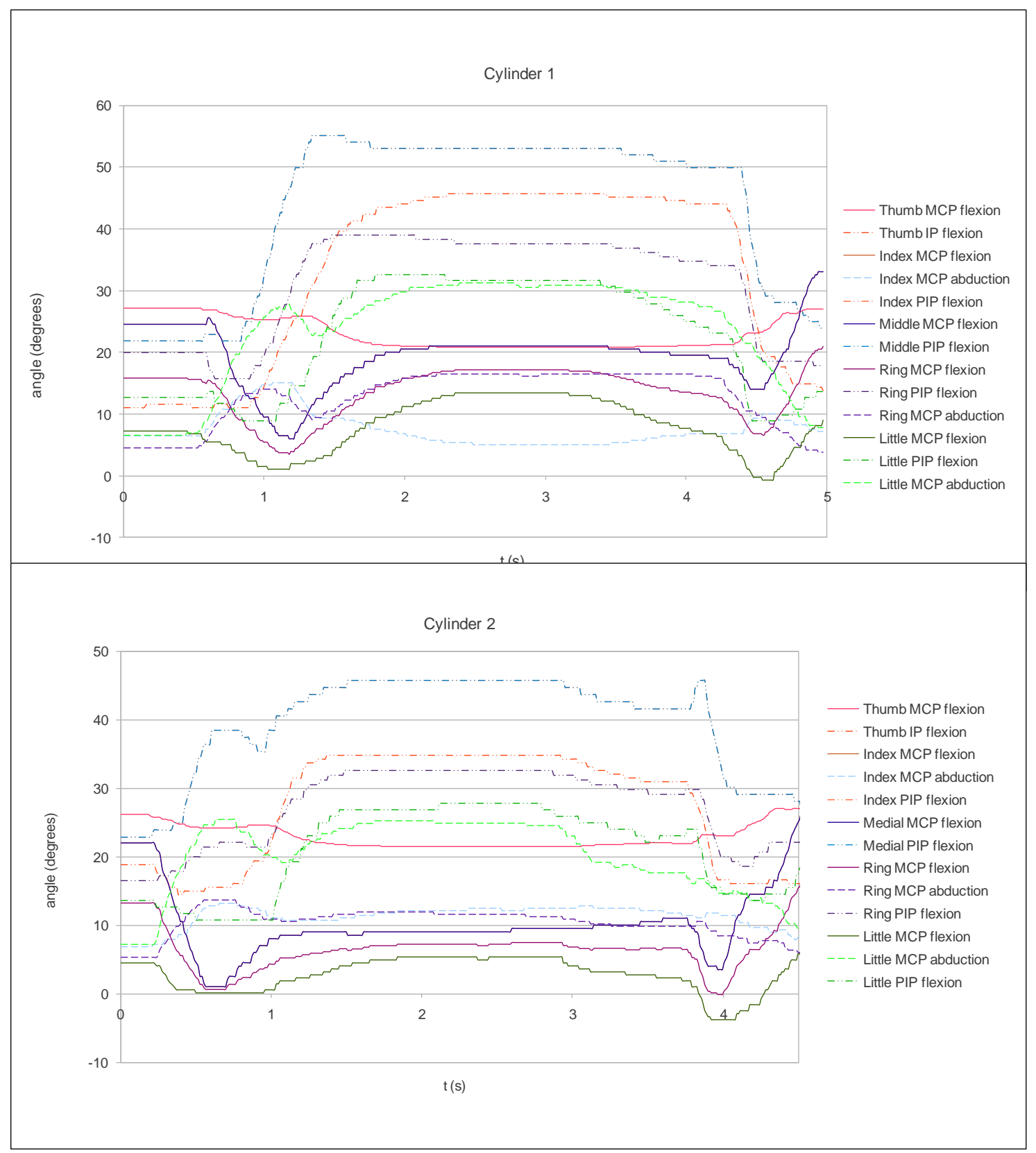

Figure 7. Joint angles (in degrees) registered during one of the repetitions for grasping both cylinders 1 and 2 .

For each cylinder, the model needs the hand most fully-open posture and the hand grasping posture (as tentative) to calculate the joint rotation rates. The most fullyopen postures (Table 2) were obtained as the mean of the most open postures identified at each of the repetitions, being the standard deviation (SD) of the joint angles lower than 8.5 degrees. For each repetition, the mean postures during the static hold of the cylinder were also obtained. The mean of these values for each cylinder was used to 
define the tentative grasping posture required to calculate the joint rotation rates (Table 3). Again, the standard deviation of the joint angles among repetitions was lower than 8.5 degrees, which indicates that the experiment was repeatable. This makes it possible to interrelate the posture data and the force data registered in different repetitions.

Table 2. Mean (SD) joint angles defining the most open postures for grasping both cylinders 1 and 2.

\begin{tabular}{|c|c|c|c|c|c|c|c|}
\hline & \multicolumn{2}{|c|}{$\mathrm{MCC}$} & \multicolumn{2}{|c|}{ MCP } & \multirow{2}{*}{$\begin{array}{c}\text { PIP } \\
\text { Flexion } \\
\left(^{\circ}\right)\end{array}$} & \multirow{2}{*}{$\begin{array}{c}\text { DIP } \\
\text { Flexion } \\
\left({ }^{\circ}\right)\end{array}$} \\
\hline & & $\begin{array}{c}\text { Flexion } \\
\left({ }^{\circ}\right)\end{array}$ & $\begin{array}{l}\text { Abduction } \\
\left({ }^{\circ}\right)\end{array}$ & $\begin{array}{c}\text { Flexion } \\
\left({ }^{\circ}\right)\end{array}$ & $\begin{array}{l}\text { Abduction } \\
\left({ }^{\circ}\right)\end{array}$ & & \\
\hline \multirow{5}{*}{ 㐫 } & Thumb & $-8.0(2.3)$ & $49.0(5.1)$ & $25.1(0.6)$ & $0.0(0.1)$ & $19.1(8.5)$ & - \\
\hline & Index & - & - & $10.2(3.5)$ & $8.9(3.2)$ & $37.6(7.9)$ & $18.8(3.9)$ \\
\hline & Medial & - & - & $10.2(3.5)$ & $0.0(0.0)$ & $37.6(7.9)$ & $18.8(3.9)$ \\
\hline & Ring & $0.0(0.5)$ & - & $6.2(1.8)$ & $9.8(2.6)$ & $22.8(6.6)$ & $11.4(3.3)$ \\
\hline & Little & $0.0(1.0)$ & - & $2.1(0.7)$ & $17.7(4.5)$ & $9.4(3.8)$ & $4.7(1.9)$ \\
\hline \multirow{5}{*}{ 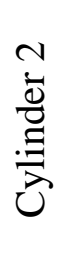 } & Thumb & $-10.0(3.1)$ & $50.0(4.6)$ & $23.9(0.3)$ & $0.0(0.1)$ & $17.6(3.3)$ & - \\
\hline & Index & - & - & $2.4(2.5)$ & $10.0(1.6)$ & $38.1(8.0)$ & $19.0(4.0)$ \\
\hline & Medial & - & - & $2.4(2.5)$ & $0.0(0.0)$ & $38.1(8.0)$ & $19.0(3.9)$ \\
\hline & Ring & $0.0(1.2)$ & - & $1.6(1.6)$ & $11.6(0.8)$ & $24.2(3.9)$ & $12.1(2.0)$ \\
\hline & Little & $0.0(0.7)$ & - & $0.9(0.8)$ & $19.5(2.1)$ & $11.7(2.2)$ & $5.8(2.9)$ \\
\hline
\end{tabular}

Table 3. Mean (SD) joint angles defining the grasping postures for both cylinders 1 and 2 .

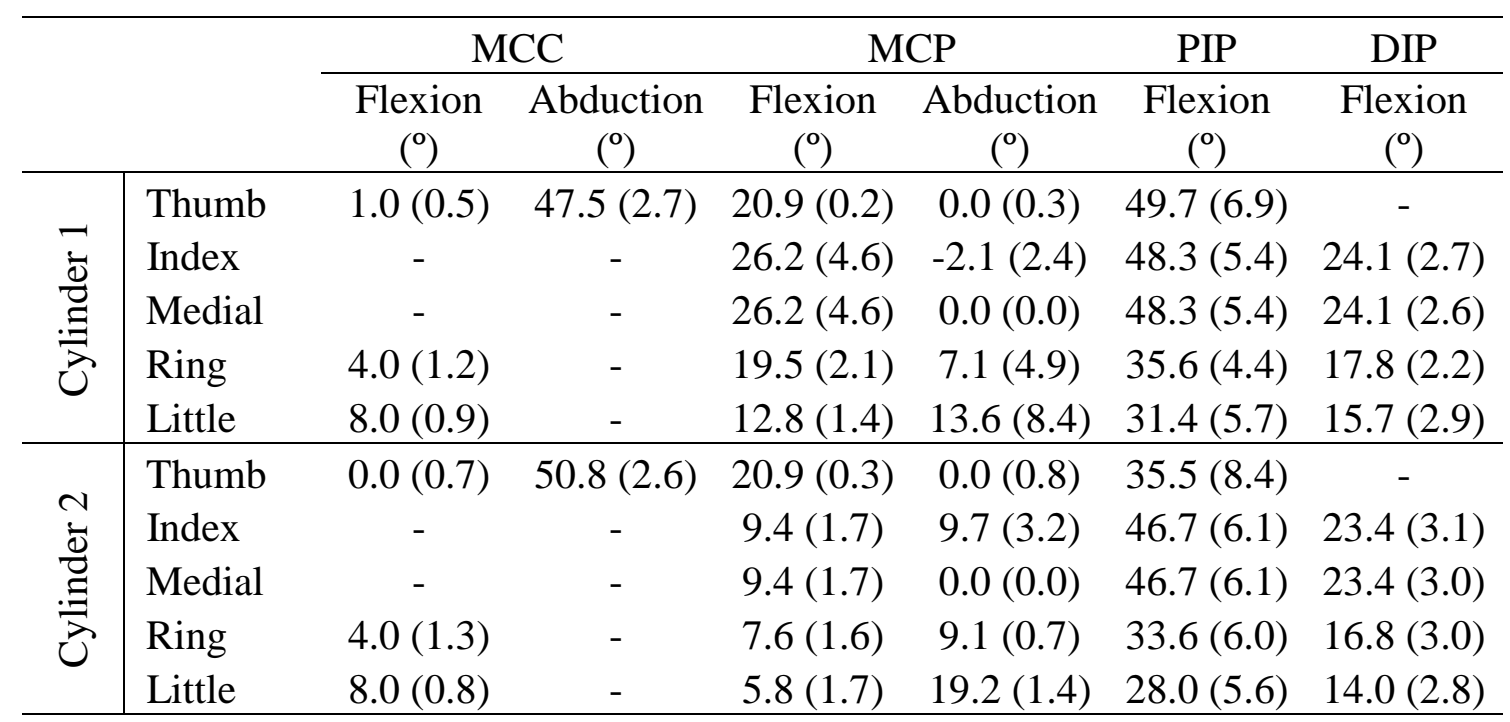

Table 4 presents the joint angles calculated by the model (from the use of the collision detection algorithm) for the grasping postures of cylinders 1 and 2 , 
respectively. They are similar to the ones measured, but not identical. In the future, it is the aim of the authors that both input postures required by the model to generate this grasping posture will be obtained by using a neural network (Kyota and others 2005; Rezzoug and Gorce 2008). Figure 8 shows the realistic appearance of the estimated grasping posture for cylinder 1.

Table 4. Grasping postures estimated by the model for both cylinders 1 and 2

\begin{tabular}{|c|c|c|c|c|c|c|c|}
\hline & \multicolumn{2}{|c|}{ MCC } & \multicolumn{2}{|c|}{ MCP } & \multirow{2}{*}{$\begin{array}{c}\text { PIP } \\
\text { Flexion } \\
\left(^{\circ}\right)\end{array}$} & \multirow{2}{*}{$\begin{array}{c}\text { DIP } \\
\begin{array}{c}\text { Flexion } \\
\left({ }^{\circ}\right)\end{array}\end{array}$} \\
\hline & & $\begin{array}{c}\text { Flexion } \\
\left({ }^{\circ}\right)\end{array}$ & $\begin{array}{l}\text { Abduction } \\
\left({ }^{\circ}\right)\end{array}$ & $\begin{array}{c}\text { Flexion } \\
\left({ }^{\circ}\right)\end{array}$ & $\begin{array}{c}\text { Abduction } \\
\left({ }^{\circ}\right)\end{array}$ & & \\
\hline \multirow{5}{*}{ 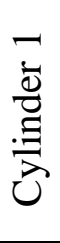 } & Thumb & 0.3 & 47.7 & 21.2 & 0.0 & 47.3 & - \\
\hline & Index & - & - & 20.4 & 1.9 & 44.4 & 18.8 \\
\hline & Medial & - & - & 23.0 & 0.0 & 46.1 & 27.4 \\
\hline & Ring & 4.2 & - & 20.0 & 7.0 & 36.2 & 43.9 \\
\hline & Little & 8.5 & - & 13.5 & 13.4 & 32.8 & 7.8 \\
\hline \multirow{5}{*}{ 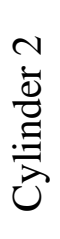 } & Thumb & -3.0 & 50.5 & 21.8 & 0.0 & 30.2 & - \\
\hline & Index & - & - & 9.7 & 9.7 & 47.1 & 23.5 \\
\hline & Medial & - & - & 12.9 & 0.0 & 51.1 & 25.5 \\
\hline & Ring & 6.7 & - & 11.6 & 7.4 & 40.0 & 20.0 \\
\hline & Little & 7.8 & - & 5.7 & 19.2 & 27.6 & 13.8 \\
\hline
\end{tabular}
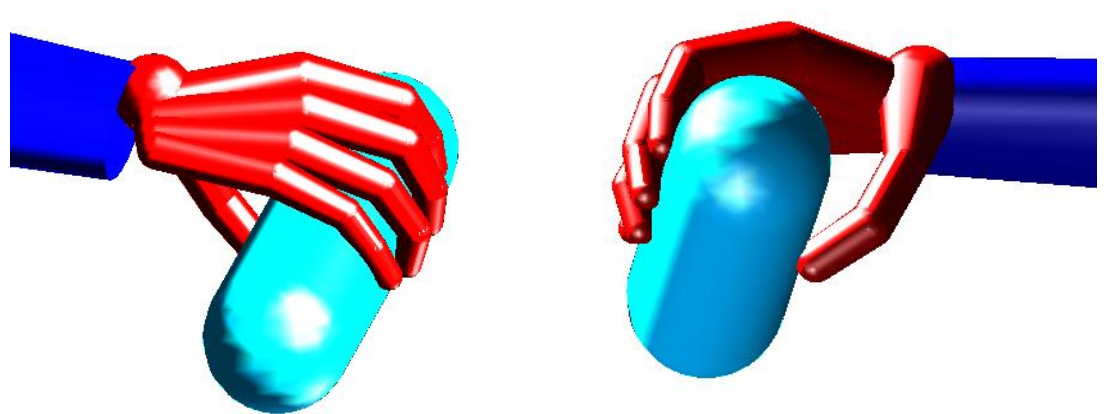

Figure 8 . Grasping posture estimated by the model for cylinder 1 .

The finger force patterns registered during the experiment can be observed in figure 9. This figure shows the forces registered by the Finger TPS ${ }^{\circledR}$ system in one of the repetitions for cylinders 1 and 2 . Due to the greater weight of cylinder 1 , it is observed a peak in the finger forces during the cylinder elevation phase corresponding to inertial effects, which is not observed for the case of cylinder 2. Finger forces 
registered during the static hold of the cylinders remain quite constant. For each repetition, the mean of the finger force registered during the static hold has been considered. The mean of the finger forces among repetitions for both cylinders are shown in table 5, along with the contact forces estimated by the model and three different modifications described later. It has to be noted that the forces registered for the thumb and the index finger varied greatly among repetitions. This confirms that there is not a unique combination of forces for grasping the cylinder, but that different safety margins can be applied for avoiding the slippery of the object. Experimental mean contributions of the thumb and fingers to the grasp force are presented in table 6 , along with the estimated ones obtained from the model and its modifications. These contributions were calculated with respect to the sum of the contact grasping forces. The experimental contributions allow identifying the thumb as the major contributor to the grasping force. 


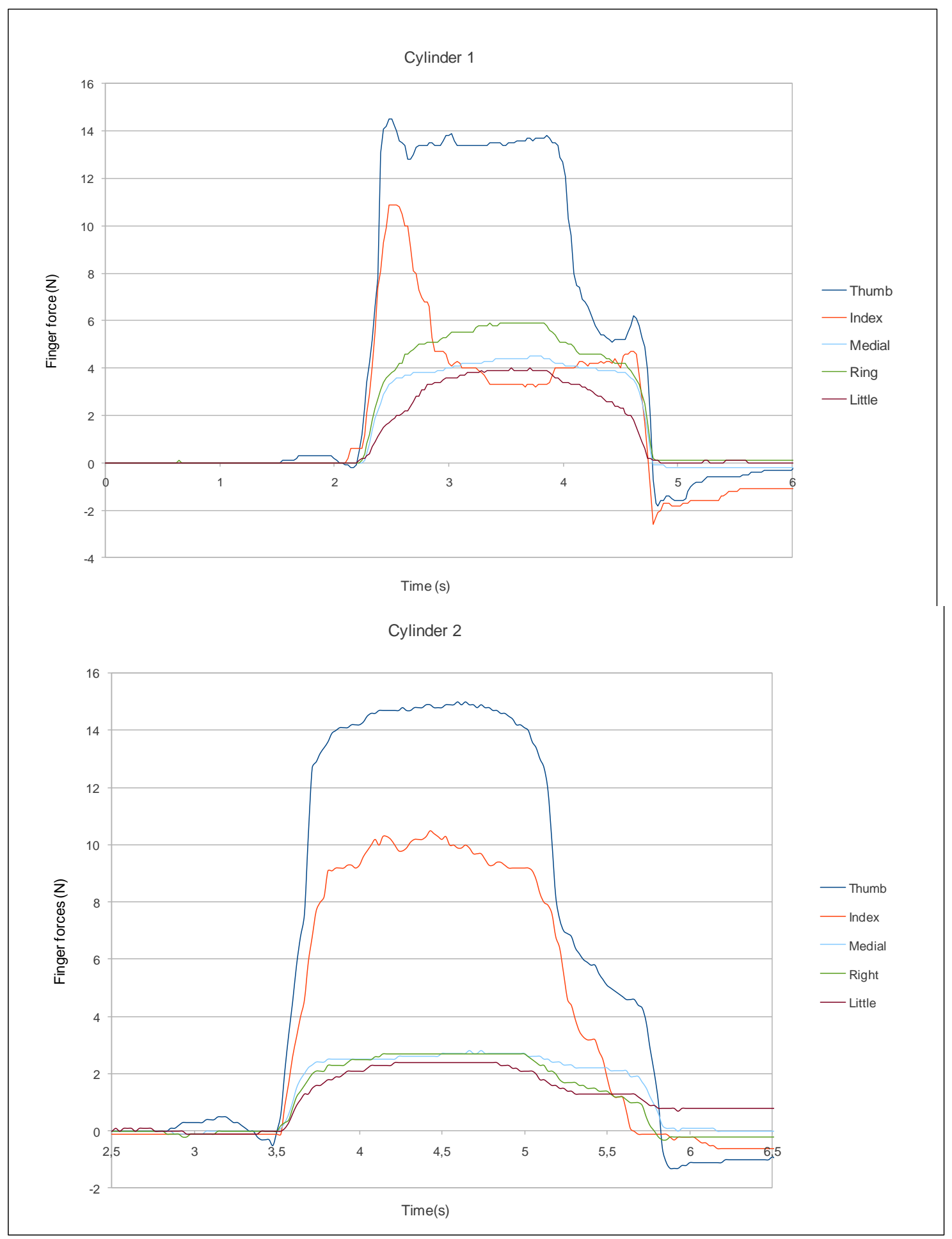

Figure 9. Finger forces $(\mathrm{N})$ registered during one of the repetitions for both cylinders 1 and 2. 
Table 5. Mean (SD) finger forces registered for the grasping of both cylinders and contact forces estimated by the model and its modifications.

\begin{tabular}{|c|c|c|c|c|c|c|c|c|}
\hline & & & Thumb & Index & Medial & Ring & Little & Total \\
\hline \multirow{13}{*}{ 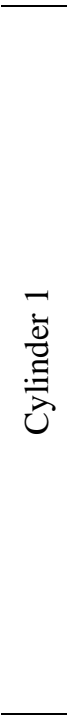 } & Experimental & Normal (N) & $10.7(4.5)$ & $3.4(2.4)$ & $4.6(0.8)$ & $5.8(0.6)$ & $4.1(1.1)$ & $28.7(3.2)$ \\
\hline & \multirow{3}{*}{ Estimated 1} & Normal (N) & 4.05 & 0.01 & 1.97 & 0.96 & 0.60 & 7.59 \\
\hline & & Tangential (N) & 3.23 & 0.01 & 1.57 & 0.76 & 0.48 & \\
\hline & & Torque $(\mathrm{N} \cdot \mathrm{mm})$ & -4.70 & 0.00 & -1.40 & -0.60 & -0.30 & \\
\hline & \multirow{3}{*}{ Estimated 2} & Normal (N) & 10.59 & 2.96 & 1.98 & 2.54 & 3.12 & 21.19 \\
\hline & & Tangential (N) & 3.18 & 0.89 & 0.59 & 0.76 & 0.94 & \\
\hline & & Torque $(\mathrm{N} \cdot \mathrm{mm})$ & 0.00 & 0.10 & -0.30 & -0.50 & -0.70 & \\
\hline & \multirow{3}{*}{ Estimated 3} & Normal (N) & 3.81 & 0.91 & 0.92 & 0.92 & 0.92 & 7.48 \\
\hline & & Tangential (N) & 3.02 & 0.73 & 0.72 & 0.71 & 0.73 & \\
\hline & & Torque $(\mathrm{N} \cdot \mathrm{mm})$ & -5.80 & 0.10 & -1.10 & -1.70 & 0.00 & \\
\hline & \multirow{3}{*}{ Estimated 4} & Normal (N) & 6.62 & 2.12 & 1.64 & 1.47 & 2.12 & 13.97 \\
\hline & & Tangential (N) & 3.15 & 1.68 & 1.27 & 0.95 & 1.69 & \\
\hline & & Torque $(\mathrm{N} \cdot \mathrm{mm})$ & -25.20 & 3.00 & -3.80 & -7.60 & 1.20 & \\
\hline \multirow{13}{*}{ 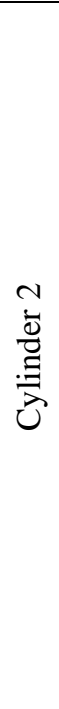 } & Experimental & Normal (N) & $1011.0(5.8)$ & $9.3(4.5)$ & $3.0(0.8)$ & $2.9(0.6)$ & $1.7(0.5)$ & $27.8(10.5)$ \\
\hline & \multirow{3}{*}{ Estimated 1} & Normal (N) & 0.41 & 0.00 & 0.24 & 0.11 & 0.01 & 0.77 \\
\hline & & Tangential (N) & 0.30 & 0.01 & 0.19 & 0.09 & 0.01 & \\
\hline & & Torque $(\mathrm{N} \cdot \mathrm{mm})$ & 1.00 & 0.00 & -0.10 & 0.00 & 0.00 & \\
\hline & \multirow{3}{*}{ Estimated 2} & Normal (N) & 0.98 & 0.18 & 0.53 & 0.00 & 0.26 & 1.95 \\
\hline & & Tangential (N) & 0.29 & 0.05 & 0.16 & 0.00 & 0.08 & \\
\hline & & Torque $(\mathrm{N} \cdot \mathrm{mm})$ & -0.10 & 0.00 & 0.00 & 0.00 & 0.00 & \\
\hline & \multirow{3}{*}{ Estimated 3} & Normal (N) & 0.45 & 0.12 & 0.11 & 0.11 & 0.11 & 0.9 \\
\hline & & Tangential (N) & 0.35 & 0.09 & 0.09 & 0.07 & 0.09 & \\
\hline & & Torque $(\mathrm{N} \cdot \mathrm{mm})$ & -0.60 & 0.00 & 0.10 & 0.30 & 0.00 & \\
\hline & \multirow{3}{*}{ Estimated 4} & Normal (N) & 4.98 & 1.79 & 1.32 & 0.48 & 1.56 & 10.13 \\
\hline & & Tangential (N) & 0.36 & 0.20 & 0.30 & 0.38 & 0.70 & \\
\hline & & Torque $(\mathrm{N} \cdot \mathrm{mm})$ & -36.50 & 16.20 & -10.90 & 0.90 & -8.70 & \\
\hline
\end{tabular}

Table 6. Mean finger force contribution (\%) registered for the grasping of both cylinders and estimated by the model and its modifications.

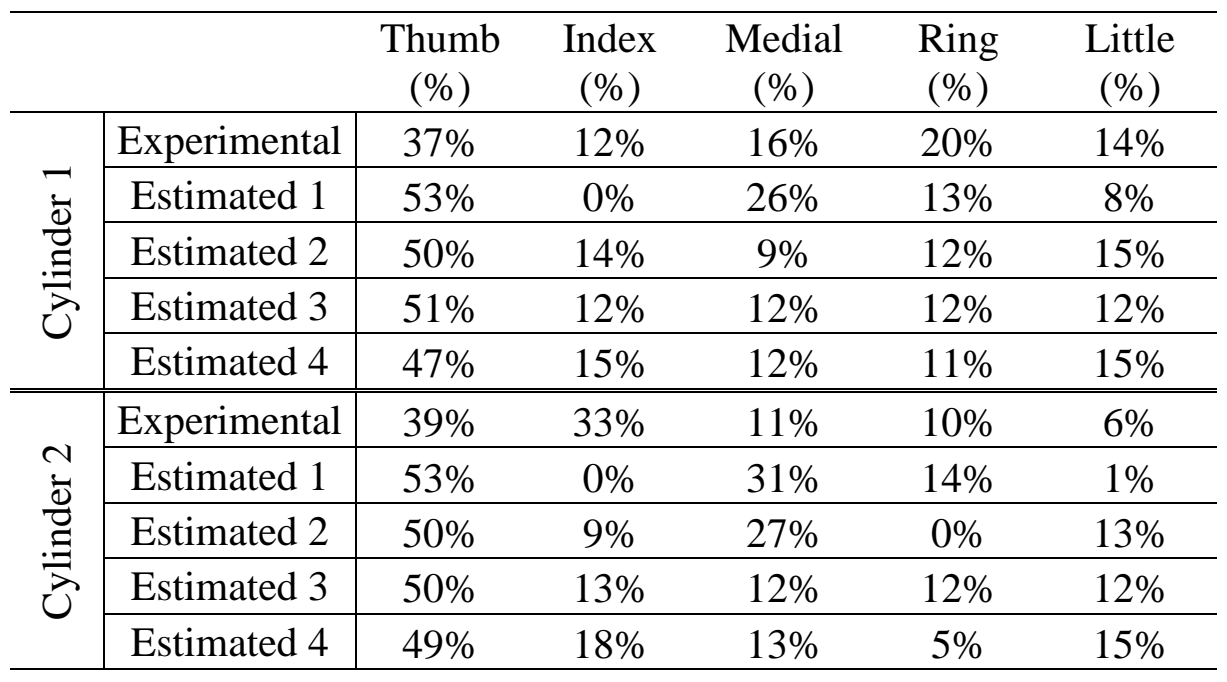


Tables 5 and 6 compare the experimental finger forces registered during the static hold of the cylinders to the contact forces and torques estimated by the model and three different modifications. The original model ('Estimated 1') considered the minimisation of the sum of the squared muscle stresses (maximisation of endurance) to solve the indeterminate problem, and a friction coefficient of 0.8 , obtained from Savescu et al. (2008). The disagreement between the experimentally measured normal forces and the estimated ones is evident. On the one hand, the estimated values are lower than the experimental ones, being the total grasping force underestimated by a $73 \%$ in the case of holding the cylinder 1 , and by a $97 \%$ in the case of holding the cylinder 2 (the lightest one). On the other hand, the estimated grasping force distributions among fingers do not match the ones measured experimentally. In particular, the model predicts that index and little fingers do not contribute at all to the grasp, which does not match the real behaviour of the human hand.

First modification to the model ('Estimated 2') tries to investigate whether an improper friction coefficient between the hand and the cylinder could be responsible for the low level of the forces estimated by the model, given that a smaller friction coefficient will demand greater normal forces to assure the grasp stability. Taking into account the data reported by Savescu et al. (2008), the effect of changing this coefficient to the very low value of 0.3 has been checked (Tables 5 and 6). Although the model estimates greater normal forces, the total grasping force estimated by the model for grasping the lighter cylinder is still far from the registered one. Furthermore, a disagreement in the force contributions of the fingers remains, especially for the lighter cylinder. This seems to indicate that the underestimated results given by the original 
model are not due to inaccuracies in the friction coefficient between the object and the hand.

The results from the simulations performed with the original model ('Estimated 1') seem to indicate that it is mathematically feasible to grasp the cylinders without the contribution of some fingers. And even that this fact could be more efficient in some aspects (maximising the endurance). However, the experimental results indicate that the CNS (central nervous system) chooses a more even distribution of the forces between fingers. Trying to account for this coordination mechanism, we have proposed the second modification of the model ('Estimated 3'). We have repeated the simulations but adding to the objective function to be minimised a term accounting for the differences between the finger forces:

$$
O B J=\sum_{i=1}^{l}\left(\frac{F_{i}}{P C S A_{i}}\right)^{2}+100^{2} \sum_{r, s=1}^{4}\left(F_{n_{r}}-F_{n_{s}}\right)^{2},
$$

where $F_{n_{r}}$ is the normal component of the contact force developed by finger $r$ and PCSA is measured in $\mathrm{cm}$. The factor $100^{2}$ has been introduced to make both combined functions comparable: we want to make comparable the muscles stresses (approximately in the range of $10 \mathrm{~N} / \mathrm{cm} 2$ ) to differences of forces of $0.1 \mathrm{~N}$. The results from these simulations are also presented in tables 5 and 6 . The use of this function allows achieving more balanced estimations of forces, but the magnitudes of the estimated forces are still lower than the experimental ones. The use of this objective function and the reduction of the friction coefficient to 0.3 (results not shown for brevity) provided a quite close estimation of forces for the heaviest cylinder, but the magnitudes of the estimated forces for the lightest cylinder were still too small compared to the experimental results. 
All these results seem to point out that the criterion that the CNS uses to select the grasping force distribution among fingers is not only related to some energetic minimisation, as the experimental forces registered are much bigger than those theoretically required to perform the grasp. The key must lie in some other factor. It is well known that humans exert grip forces taking a force safety margin into consideration to improve the grasp stability (Jenmalm et al., 1998). In this sense, in robotics, the selection of the grasp to be executed by a robotic hand is performed by calculating different kinds of grasp quality measures. Many different quality measure definitions can be found in the robotics literature. Most of them are related to the capability of handling the object once grasped or the ability of the grasp to resist external disturbances (stability). This knowledge might be used also for studying the human grasp. For the experiment simulated in this work, it might make sense that the CNS would try to ensure certain level of stability of the cylinder being grasped, given that the subject was asked to hold it still during some seconds. The third modification of the original model ('Estimated 4') has been performed to simulate this criterion.

Most of the robotic quality measures that evaluate the stability of the grasps are geometrical measures that only take into account the contact points and the directions of the normal contact forces. These measures do not account for the magnitudes of the forces and would not be useful for defining the objective function in the case under study. Obviously, the sum of the components of the applied forces that are normal to the object boundary is indicative of the force efficiency in the grasp. Then, a quality measure can be defined as the inverse of the sum of the magnitudes of the normal components of the applied forces required to balance an expected demanding wrench (Liu and others 2004). The index must be minimised to get an optimum grasp. 
The results of minimising that function ('Estimated 4') that looks for a more stable grasp, are also shown in tables 5 and 6 . The magnitudes of the forces estimated by the model with this assumption are much closer to the experimental ones than with any other of the previous objective functions, even for the lighter cylinder. These results confirm that, for the experiment being simulated, the CNS is trying to ensure the stability of the grasped cylinder. Although the results do not match exactly the experimental measurements, they adjust better than any other of the previously considered scenarios.

Anyway, the criterion selected by the CNS in each case should probably be a function of the task to be performed. The objective function that has provided good results in these simulations may not provide so good results under other requirements. For example, if the subject were asked to grasp a cylindrical bottle to pour water. In that case, the grasp should allow certain level of manipulability that will be in conflict with the stability. More research is needed in this matter. In any case, what seems clear is that the popular objective function sum of the squared muscle stresses is not suitable for grasping simulation using biomechanical models of the hand, or that it should be at least complemented by task dependent grasp quality measures (manipulability or stability).

\section{Conclusions}

The extension of a previously validated biomechanical model of the hand to study the human grasp has been presented. The geometrical representation of the hand segments and the grasped object as a spherical extension of polytopes (s-topes) has shown a sufficient level of realism and a fast and efficient collision detection.

Realistic grasping postures have been obtained through the use of the grasping algorithm implemented in the model. However, the generation of the natural grasping 
motion path of the hand from a fully opened state to a clenched one required the calculation of rotation rates at each joint from two experimentally measured postures (the most fully opened posture and the clenched posture). To avoid these experimental input data, more research is required in the future to develop a neural network able to obtain both input postures required by the model.

Using the contact information provided by the grasping algorithm, the equilibrium of the grasped object has been added to the model through the consideration of a simple soft contact model that considers the frictional moment at each contact zone. That has leaded to an indeterminate problem that has been solved by minimising different objective functions. The model underestimated the normal contact forces when the criterion of minimising the sum of the squared muscle stresses was used. Furthermore, according to the model predictions, it is mathematically feasible to grasp the cylinders without the contribution of some fingers, and this is more efficient in some aspects. But it is not the real behaviour of the human hand that was experimentally observed.

For the simulated experiment, best results were obtained when the indeterminate problem was solved using a robotic grasp quality measure as objective function that tried to ensure the stability of the grasped cylinder. Although this function has provided good results in these simulations, it may fail for others entailing certain level of manipulability, as the criterion selected by the CNS in each case will be probably a function of the task to be performed. Further research on the application of other robotics grasp quality measures for different tasks involving different levels of stability and manipulability is needed.

Therefore, main contribution of the paper is showing that the widespread Crowninshield minimisation function does not work well when trying to simulate the 
grasping of an object with an already validated 3D model of the hand. And that the consideration of stability criteria improves the estimations. This result is significant in the context of human grasp modelling and has not been reported previously in the literature, contributing to a better understanding of human grasp.

Finally, the model presented in this work has been used to study only grasps of cylinders with the fingertips. More complex grasps, involving more contact zones and more complex object geometries should be investigated in the future.

\section{Acknowledgements}

We are grateful for the financial support of the Fundació Caixa-Castelló and the Universitat Jaume I throughout the project P1-1B2009-40; of the Spanish Research and Innovation Ministry, and the EU (FEDER funds) throughout the project DPI201018177; and of European Community's Seventh Framework Programme through the project GRASP, IST-FP7-IP-215821. This research is related to these three projects.

\section{Nomenclature}

3D Three-dimensional

ADD Adductor pollicis

ADQ Abductor digiti quinti

APB Abductor pollicis brevis

APL Abductor pollicis longus

CE Contractile element

CMC Carpometacarpal

CNS Central nervous system

DI Dorsal interosseous

DIP Distal interphalangeal

DOF Degrees of freedom

EDC Extensor digitorum communis

EDQ Extensor digiti quinti

EGP Experimental grasping posture

EI Extensor indicis

EPB Extensor pollicis brevis

EPL Extensor pollicis longus

FDQ Flexor digiti quinti 


$\begin{array}{ll}\text { FP } & \text { Flexor profundus } \\ \text { FPB } & \text { Flexor pollicis brevis } \\ \text { FPL } & \text { Flexor pollicis longus } \\ \text { FS } & \text { Flexor superficialis } \\ \text { HB } & \text { Hand breadth } \\ \text { HL } & \text { Hand length } \\ \text { IP } & \text { Interphalangeal } \\ \text { LU } & \text { Lumbrical } \\ \text { MCP } & \text { Metacarpophalangeal } \\ \text { MOP } & \text { Most open posture } \\ \text { OPP } & \text { Opponens pollicis } \\ \text { PCSA } & \text { Physiological cross-sectional area } \\ \text { PEE } & \text { Parallel elastic element } \\ \text { PIP } & \text { Proximal interphalangeal } \\ \text { SD } & \text { Standard deviation } \\ \text { SEE } & \text { Series elastic element } \\ \text { VI } & \text { Volar interosseous }\end{array}$

\section{References}

An KN, Chao EYS, Kaufman KR. 1991. Basic orthopaedics biomechanics. New York: Raven Press. Ltd. Analysis of muscle and joint loads; p.1-50.

An K, Chao E, Cooney W, Linscheid R. 1979. Normative model of human hand for biomechanical analysis. J Biomech 12(10):775-788.

Bernabeu E, Tornero J. 2002. Hough transform for distance computation and collision avoidance. IEEE Trans Rob Autom 18(3):393-398.

Biryukova EV, Yourovskaya VZ. 1994. Advances in the biomechanics of the hand and wrist. New York: Plenum Press. A model of human hand dynamics; p. 107-122.

Brand PW, Hollister AM. 1992. Clinical mechanics of the hand. Third ed. St. Louis: Elsevier Science Health Science div.

Buchholz B, Armstrong T, Goldstein S. 1992. Anthropometric data for describing the kinematics of the human hand. Ergonomics 35(3):261-273.

Buchner H, Hines M, Hemami H. 1988. A dynamic-model for finger interphalangeal coordination. J Biomech 21(6):459-468.

Casolo F, Lorenzi V. 1994. Advances in the biomechanics of the hand and wrist. New York: Plenum Press. Finger mathematical modelling and rehabilitation; p. 197-223.

Chao EY, An KN. 1978. Determination of internal forces in human hand. ASCE J Eng Mech Div 104(1):255-272.

Chao EY, Opgrande JD, Axmear FE. 1976. Three dimensional force analysis of finger joints in selected isometric hand functions. J Biomech 9(6):387. 
Choi J. 2008. Developing a 3-dimensional kinematic model of the hand for ergonomic analyses of hand posture, hand space envelope, and tendon excursion. University of Michigan.

Ciocarlie M, Lackner C, Allen P. 2007. Soft finger model with adaptive contact geometry for grasping and manipulation tasks. Paper presented at: World Haptics 2007. Second Joint EuroHaptics Conference, 2007 and Symposium on Haptic Interfaces for Virtual Environment and Teleoperator Systems.

Ciocarlie M, Miller A, Allen P. 2005. Grasp analysis using deformable fingers. Paper presented at: IEEE/RSJ International Conference on Intelligent Robots and Systems.

Crowninshield R, Brand R. 1981. A physiologically based criterion of muscle force prediction in locomotion. J Biomech 14(11):793-801.

Endo Y, Kanai S, Kishinami T, Miyata N, Kouchi M, Mochimaru M. 2007. Virtual grasping assessment using 3D digital hand model. Presented at: 10th Annual Applied Ergonomics Conference: Celebrating the Past - Shaping the Future.

Esteki A, Mansour JM. 1997. A dynamic model of the hand with application in functional neuromuscular stimulation. Ann Biomed Eng 25(3):440-51.

Fok KS, Chou SM. 2010. Development of a finger biomechanical model and its considerations. J Biomech 43(4):701-13.

García de Jalón J, Bayo E. 1994. Kinematic and dynamic simulation of multibody systems. New York: Springer-Verlag.

Gilbert E, Johnson D, Keerthi S. 1988. A fast procedure for computing the distance between complex objects in 3-dimensional space. IEEE J Robotic Autom 4(2):193-203. Giurintano D, Hollister A, Buford W, Thompson D, Myers L. 1995. A virtual 5-link model of the thumb. Med Eng Phys 17(4):297-303.

Goldstein S, Armstrong T, Chaffin D, Matthews L. 1987. Analysis of cumulative strain in tendons and tendon sheaths. J Biomech 20(1):1-6.

Hajian A, Howe R. 1997. Identification of the mechanical impedance at the human finger tip. J Biomech Eng-T ASME 119(1):109-114.

Hatze H. 1981. Myocibernetic control models of skeletal muscle. Pretoria: University of South Africa.

Hill A. 1938. The heat of shortening and the dynamic constants of muscle. Proceedings of the Royal Society of London Series B-Biological Sciences 126(843):136-95.

Hollister A, Giurintano D, Buford W, Myers L, Novick A. 1995. The axes of rotation of the thumb interphalangeal and metacarpophalangeal joints. Clin Orthop (320):188-93. 
Howe R, Kao I, Cutkosky M. 1988. Sliding of robot fingers under combined tornsion and shear loading. Presented at: IEEE Int. Conf. on Robotics and Automation.

Howe R, Cutkosky M. 1996. Practical force-motion models for sliding manipulation. Int J Robotics Res 15(6):557-72.

Jacobson M, Raab R, Fazeli B, Abrams R, Botte M, Lieber R. 1992. Architectural design of the human intrinsic hand muscles. J Hand Surg-Am Volume 17A(5):804-9. Jenmalm P, Goodwin AW and Johansson RS. 1998. Control of grasp stability when humans lift objects with different surface curvatures, J. Neurophysiol 79:1643-1652.

Johnson KL. 1985. Contact mechanics. Cambridge University Press.

Kamper D, Fischer H, Cruz E. 2006. Impact of finger posture on mapping from muscle activation to joint torque. Clin Biomech 21(4):361-9.

Kao I, Linch K, Burdick JW. 2008. Springer-Verlag. Handbook of robotics. Chapter 27, Contact modeling and manipulation; p. 647-668

Kao I, Yang F. 2004. Stiffness and contact mechanics for soft fingers in grasping and manipulation. IEEE Trans Rob Autom 20(1):132-5.

Kao I, Cutkosky M. 1992. Quasi-static manipulation with compliance and sliding. Int J Robotics Res 11(1):20-40.

Kapandji AI. 1998. Fisiologie articulaire. membre supérieur. Paris: Editions Maloine.

Kaufman K, An K, Litchy W, Chao E. 1991. Physiological prediction of muscle forces .1. theoretical formulation. Neuroscience 40(3):781-92.

Kurita Y, Onoue T, Ikeda A, Ogasawara T. 2009. Biomechanical analysis of subjective pinching effort based on tendon-skeletal model. Conf Proc IEEE Eng Med Biol Soc. 2009; p. 5231-5234.

Kyota F, Watabe T, Saito S, Nakajima M. 2005. Detection and evaluation of grasping positions for autonomous agents. Presented at: International Conference on Cyberworlds, 2005; Singapore.

Lee JW, Rim K. 1990. Maximum finger force prediction using a planar simulation of the middle finger. Proc Inst Mech Eng Part H J Eng Med 204:169-78.

Lee SW, Chen H, Towles JD, Kamper DG. 2008a. Effect of finger posture on the tendon force distribution within the finger extensor mechanism. J Biomech Eng-T ASME 130(5):051014.

Lee SW, Chen H, Towles JD, Kamper DG. 2008b. Estimation of the effective static moment arms of the tendons in the index finger extensor mechanism. J Biomech 41(7):1567-73. 
Leijnse J And Kalker J. 1995. A 2-dimensional kinematic model of the lumbrical in the human finger. J Biomech 28(3):237-49.

Leijnse J, Bonte J, Landsmeer J, Kalker J, Vandermeulen J, Snijders C. 1992. Biomechanics of the finger with anatomical restrictions - the significance for the exercising hand of the musician. J Biomech 25(11):1253-64.

Lemay M, Crago P. 1996. A dynamic model for simulating movements of the elbow, forearm, and wrist. J Biomech 29(10):1319-30.

Liu G, Xu J, Wang X, Li Z. 2004. On quality functions for grasp synthesis, fixture planning, and coordinated manipulation. IEEE Trans. Autom. Sci. Eng. 1(2):146-62.

Mansour JM, Rouvas C, Sarangapani J, Hendrix L, Crago PE. 1994. Advances in the biomechanics of the hand and wrist. New York: Plenum Press. Quantitative functional anatomy of finger muscles: Application to controlled grasp; p. 177-188.

Mora MC., Sancho-Bru JL, Iserte JL, Pérez-González A, Porcar-Bonet B. 2011. Protocolo de calibración para guante instrumentado en la caracterización cinemática del agarre humano. Presented at: X Congreso Iberoamericano de Ingeniería Mecánica; Porto, Portugal.

Qiu D, Fischer HC, Kamper DG. 2009. Muscle activation patterns during force generation of the index finger. Conf Proc IEEE Eng Med Biol Soc. 2009; p. 3987-3990.

Rezzoug N, Gorce P. 2008. Prediction of fingers posture using artificial neural networks. J Biomech 41(12):2743-9.

Roa Garzón M. 2009. Grasp planning methodology for 3D arbitrary shaped objects. Universitat Politècnica de Catalunya.

Roloff I, Schoffl V, Vigouroux L, Quaine F. 2006. Biomechanical model for the determination of the forces acting on the finger pulley system. J Biomech 39(5):915-23. Sancho-Bru JL, Giurintano DJ, Pérez-González A, Vergara M. 2003a. Optimum tool handle diameter for a cylinder grip. J Hand Ther 16(4):337-42.

Sancho-Bru JL, Perez-Gonzalez A, Vergara M, Giurintano DJ. 2003b. A 3D biomechanical model of the hand for power grip. J Biomech Eng 125(1):78-83.

Sancho-Bru JL, Perez-Gonzalez A, Vergara-Monedero M, Giurintano D. 2001. A 3-D dynamic model of human finger for studying free movements. J Biomech 34(11):1491500 .

Sancho-Bru JL, Vergara M, Rodriguez-Cervantes P, Giurintano DJ, Perez-Gonzalez A. 2008. Scalability of the muscular action in a parametric 3D model of the index finger RID E-6162-2010 RID C-8708-2011. Ann Biomed Eng 36(1):102-7. 
Savescu AV, Latash ML, Zatsiorsky VM. 2008. A technique to determine friction at the fingertips. J Appl Biomech 24(1):43-50.

Smith E, Pearson J, Juvinall R, Bender L. 1964. Role of finger flexors in rheumatoid deformities of metacarpophalangeal joints. Arthritis Rheum 7(5P1):467-480.

Spoor C. 1983. Balancing a force on the fingertip of a two-dimensional finger model without intrinsic muscles. J Biomech 16(7):497-504.

Spoor C, Landsmeer J. 1976. Analysis of zigzag movement of human finger under influence of extensor digitorum tendon and deep flexor tendon. J Biomech 9(9):561-6.

Storace A, Wolf B. 1982. Kinematic analysis of the role of the finger tendons. J Biomech 15(5):391-3.

Storace A, Wolf B. 1979. Functional-analysis of the role of the finger tendons. J Biomech 12(8):575-8.

Thomas DH, Long C, Landsmeer JMF. 1968. Biomechanical consideration of lumbricalis behaviour in the human finger. $\mathrm{J}$ Biomech 1:107-15.

Valero-Cuevas FJ, Zajac FE, Burgar CG. 1998. Large index-fingertip forces are produced by subject-independent patterns of muscle excitation. J Biomech 31(8):693703.

Valero-Cuevas F. 2005. An integrative approach to the biomechanical function and neuromuscular control of the fingers. J Biomech 38(4):673-84.

Valero-Cuevas F. 2000. Predictive modulation of muscle coordination pattern magnitude scales fingertip force magnitude over the voluntary range. J Neurophysiol 83(3):1469-79.

Valero-Cuevas F, Towles J, Hentz V. 2000. Quantification of fingertip force reduction in the forefinger following simulated paralysis of extensor and intrinsic muscles. $\mathrm{J}$ Biomech 33(12):1601-9.

Vigouroux L, Quaine F, Labarre-Vila A, Moutet F. 2006. Estimation of finger muscle tendon tensions and pulley forces during specific sport-climbing grip techniques. $\mathbf{J}$ Biomech 39(14):2583-92.

Weightman B, Amis A. 1982. Finger joint force predictions related to design of joint replacements. J Biomed Eng 4(3):197-205.

Wu JZ, An K, Cutlip RG, Dong RG. 2010. A practical biomechanical model of the index finger simulating the kinematics of the muscle/tendon excursions. Biomed Mater Eng 20(2):89-97. 
Zajac F. 1989. Muscle and tendon - properties, models, scaling, and application to biomechanics and motor control. Crit Rev Biomed Eng 17(4):359-411. 